http://jmscr.igmpublication.org/home/ ISSN (e)-2347-176x ISSN (p) 2455-0450

crossref DOI: https://dx.doi.org/10.18535/jmscr/v8i2.27

\title{
Role of Virtual Colonoscopy in the Diagnosis of Colonic Pathologies
}

\author{
Author \\ Dr P Ramravi Kumar \\ Associate Professor, Department of Radiology, Prathima Institute of Medical Sciences, \\ Naganoor, Karimnagar \\ *Corresponding Author \\ Dr P Ramravi Kumar
}

\begin{abstract}
CT colonography through 128 slice MDCT provides a virtual noninvasive opportunity to visualize the Colon with very good resolution at minimum radiation, in multiple planes in both retrograde and anterograde directions through $3 D$ reconstruction software, hence lumen missed by VC behind the haustral folds can be visualized in the anterograde direction. We in the present study evaluated the ability of virtual colonoscopy to detect colorectal cancers and polyps in suspected patients of colonic pathology Methods: This prospective study was conducted in the Department of Radiology, Prahtima Institute of Medical Sciences, Naganoor, Karimnagar. Patients aged 30 to 80 yrs. complaining of colonic symptoms like constipation, melena, haematochezia, loss of weight and known cases of colonic pathology. All the patients will be given $30 \mathrm{ml}$ heptulac (lactulose) mixed in water at $9 \mathrm{pm}$ the earlier day and put them on NBM overnight which will be followed by rectal enema twice spaced 4 hours and 1 hour before the CT scan. Virtual colonoscopy $(C T-V C)$ examinations will be performed according to a standard protocol. Results: $n=32$ cases with suspected colonic pathologies $n=21$ cases had colonic findings forming $65 \%$ of the cases with positive colonic findings $48 \%$ had some kind of polyps while $29 \%$ had carcinoma colon. $N=6$ cases had carcinoma of the colon among the $N=32$ cases forming $18.7 \%$ of the cases. While $67 \%$ percent of the carcinomas were in the distal colon 33\% had carcinoma of the proximal colon. Among the 6 cases of carcinoma colon, $n=4$ cases had advancing carcinoma beyond the colonic wall. Among the $n=4$ cases 50 percent $n=2$ cases presented with metastasis to distal organs. Conclusions: Virtual colonoscopy was found to be very useful because of $3 D$ viewing of colon both in forward as well as reverse directions. However, it has limitations because it depends on proper bowel preparation. Electronic bowel cleansing or barium fecal tagging may improve sensitivity. This technique is very useful in elderly patients where sedation is contraindicated and also useful in patients with Diverticulosis where chances of perforation are high by conventional colonoscopy.

Keywords: Virtual Colonoscopy, Colonic Pathologies.
\end{abstract}

\section{Introduction}

The colon is the primary target of many functional and pathological disorders, which may have an acute and chronic presentation among which colorectal cancer (CRC) is a formidable health problem worldwide. It is the third most common cancer in men (663000 cases, $10.0 \%$ of all cancer cases) and the second most common in women 571000 cases, $9.4 \%$ of all cancer cases ${ }^{[1]}$. Almost $60 \%$ of cases are encountered in developed 
countries. The number of CRC-related deaths is estimated to be approximately 608000 worldwide, accounting for $8 \%$ of all cancer deaths and making CRC the fourth most common cause of death due to cancer. In India, the annual incidence rates (AARs) for colon cancer and rectal cancer in men are 4.4 and 4.1 per 100000 , respectively. The AAR for colon cancer in women is 3.9 per 100000 Colon cancer ranks 8 th and rectal cancer ranks 9 th among men. For women, rectal cancer does not figure in the top 10 cancers, whereas colon cancer ranks 9 th $^{[2]}$. In the 2013 report, the highest AAR in men for CRCs was recorded in Thiruvananthapuram (4.1) followed by Banglore (3.9) and Mumbai (3.7). The highest AAR in women for CRCs was recorded in Nagaland (5.2) followed by Aizwal (4.5) ${ }^{[2]}$. Most cases of CRC develop from previously benign neoplastic polyps, adenomas, according to the adenoma-carcinoma sequence concept ${ }^{[3]}$. The endoscopic removal of adenomas (secondary prevention) plus post polypectomy surveillance are associated with a substantial reduction of incidence and thus mortality from $\mathrm{CRC}^{[4-7]}$. Hence early economical screening for polyps reduces its incidence especially when the subject is not compliant. With rapid urbanization, changing food habits and sedentary lifestyles there is a likelihood of increasing incidence in India. Hence this study gives an acceptable, compliant and economically feasible opportunity, especially for the screening of susceptible populations. Hence this study plans screening motivated volunteers and patients with 128 slice MDCT available in the Hospital and interpreted based on the amount of the colon lumen distended for study and if possible screen for the pathology accordingly.

\section{Material and Methods}

This prospective study was conducted in the Department of Radiology, Prahtima Institute of Medical Sciences, Naganoor, Karimnagar. Institutional Ethical committee permission was obtained for the study as per protocol. The inclusion criteria were patients aged 30 to $80 \mathrm{yrs}$. complaining of colonic symptoms like constipation, melena, haematochezia, loss of weight and known cases of colonic pathology. Exclusion criteria were pregnant women, children and Acute abdominal conditions like intestinal obstruction, peritonitis, etc. All the patients will be given $30 \mathrm{ml}$ heptulac (lactulose) mixed in water at $9 \mathrm{pm}$ the earlier day and put them on NBM overnight which will be followed by rectal enema twice spaced 4 hours and 1 hour before the CT scan. Care was taken regarding volume loss due to diarrhea with IV fluids and blood pressure and electrolyte monitoring. The combination of stool softener also includes PEG (polyethylene glycol) and soap water depending on the availability. Bowel insufflation: Patients with prepared clean bowel will be insufflated with clean warm room air through the small rectal tube to the patient's tolerance and checking for optimum distention of the entire colon with CT scout films. CT Colonography involves air insufflation of a clean, prepared colon via a small rectal tube, thin-section CT scanning of the abdomen and pelvis in both the supine and prone positions, and interpretation of images at a reading monitor capable of two-dimensional (2D) and three-dimensional (3D) post-processing using Philips Ingenuity software. Virtual colonoscopy (CT-VC) examinations will be performed according to a standard protocol. Patients are placed in the right lateral decubitus position on the CT table and a rectal enema tube was inserted. Patients will be then turned supine and room air gently insufflated into the colon to patient tolerance. A standard CT scout film of abdomen and pelvis was acquired to assess the degree of colonic distension, and further air insufflation performed if required. Using the CT scout film, each examination is tailored to encompass the entire colon from the caecum to rectum. All CT examinations shall be performed using an MDCT scanner. Images shall be acquired using collimation of $64 \times 0.625$ with a pitch of 0.797 , $110 \mathrm{~mA}, 110 \mathrm{kVp}$, and $512 \times 512$ matrices. A single breath-hold acquisition will be used when 


\section{JMSCR Vol||08 ||Issue ||02||Page 134-139||February}

possible to encompass the entire colon. Images shall be reconstructed at $1 \mathrm{~mm}$ intervals. Following the supine scan, the helical CT will be repeated with the patient prone. Interpretation of abnormalities includes morphology and heterogenicity based on location and mobility with changing positions. While lesions greater than $5 \mathrm{~mm}$ classified as polyps including sessile and pedunculated. Any growth was studied with contrast video colonoscopy for correlation. All the cases will be seen on both 2D multiplanar and 3D navigation for interpretation. Metastasis and the local extension will be included under ECF's.

\section{Results}

Our study compresses $n=32$ cases with suspected colonic pathologies showing the following results. Most of the cases in the study group were from 40 to 60 years with the combined percentage being $65 \%$. In the study group $65 \%$ were male and $35 \%$ females.

Table 1: Study group age-wise composition of the cases

\begin{tabular}{|l|c|c|}
\hline Age & No. of cases & Percentage \\
\hline $\mathbf{3 0 - 4 0}$ & 4 & $13 \%$ \\
\hline $\mathbf{4 0 - 5 0}$ & 10 & $31 \%$ \\
\hline $\mathbf{5 0 - 6 0}$ & 11 & $34 \%$ \\
\hline $\mathbf{6 0 - 7 0}$ & 5 & $16 \%$ \\
\hline $\mathbf{7 0 - 8 0}$ & 2 & $6 \%$ \\
\hline Total & 32 & $100 \%$ \\
\hline
\end{tabular}

Graph 1: Showing the distribution of the cases of gender-wise

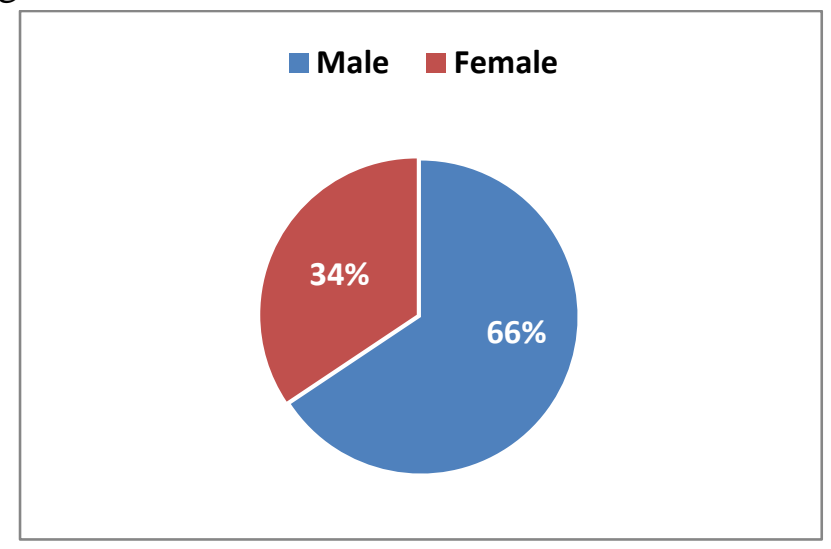

Among the $\mathrm{N}=32$ cases, $\mathrm{n}=21$ cases had colonic findings forming $65 \%$ of the cases with positive colonic findings $48 \%$ had some kind of polyps while $29 \%$ had carcinoma colon. $\mathrm{N}=6$ cases had carcinoma of the colon among the $\mathrm{N}=32$ cases forming $18.7 \%$ of the cases. While $67 \%$ percent of the carcinomas were in the distal colon $33 \%$ had carcinoma of the proximal colon. Among the 6 cases of carcinoma colon, $n=4$ cases had advancing carcinoma beyond the colonic wall. Among the $n=4$ cases 50 percent $n=2$ cases presented with metastasis to distal organs.

Table 3: Distribution of colonic lesions

\begin{tabular}{|l|c|c|c|c|}
\hline Lesions & Male & Female & $\begin{array}{c}\text { Total No. } \\
\text { of cases }\end{array}$ & Percentage \\
\hline Polyps & 6 & 4 & 10 & $48 \%$ \\
\hline Carcinoma & 3 & 3 & 6 & $29 \%$ \\
\hline Diverticulae & 3 & 1 & 4 & $19 \%$ \\
\hline Lipoma & 1 & 0 & 1 & $5 \%$ \\
\hline Total & $\mathbf{1 3}$ & $\mathbf{8}$ & $\mathbf{2 1}$ & $\mathbf{1 0 0 \%}$ \\
\hline
\end{tabular}

Table 4: Frequency of presenting symptoms

\begin{tabular}{|l|c|c|}
\hline Symptoms & No. of Cases & Percentage \\
\hline Altered bowel habits & 3 & $9 \%$ \\
\hline Constipation & 21 & $66 \%$ \\
\hline Bleeding per rectum & 4 & $13 \%$ \\
\hline Weight loss & 2 & $6 \%$ \\
\hline Abdominal discomfort & 2 & $6 \%$ \\
\hline Total & $\mathbf{3 2}$ & $\mathbf{1 0 0 \%}$ \\
\hline
\end{tabular}

Among the $n=32$ cases, $n=30$ Extra colonic findings were found with $n=4$ cases with significant findings that have to be reported. $\mathrm{N}=3$ cases of complications of malignancy and $\mathrm{N}=1$ case of incidental malignancy (Abdominal lymphoma).

Table 5: Extra colonic findings lesion wise distribution

\begin{tabular}{|l|c|c|}
\hline Lesions Types & No of Cases & Percentage \\
\hline Cysts in solid organs & 10 & $33 \%$ \\
\hline Renal and GB Calculi & 8 & $27 \%$ \\
\hline Vascular calcifications & 6 & $20 \%$ \\
\hline Lymphoma & 1 & $3 \%$ \\
\hline Hydro nephrosis & 1 & $3 \%$ \\
\hline Abdominal aortic aneurysm & 1 & $3 \%$ \\
\hline Local and distal metastasis & 3 & $10 \%$ \\
\hline Total & $\mathbf{3 0}$ & $\mathbf{1 0 0 \%}$ \\
\hline
\end{tabular}

Sodium picosulfate is a laxative was superior to PEG or Soap water achieving excellent stool cleansing for a study. The $n=8$ cases where Picosylate was used $n=7$ cases had good stool cleansing forming 87 percent. While soap water 
and PEG gives 41 percent average stool cleansing and 37 percent good stool cleansing. Among the $\mathrm{n}=32$ cases, Excellent and good distension was found in $\mathrm{n}=16(50 \%)$ of cases and Average distension was found in $n=11$ cases which are good enough for the conduction of the study.

Graph 2: Adequacy of colonic distension

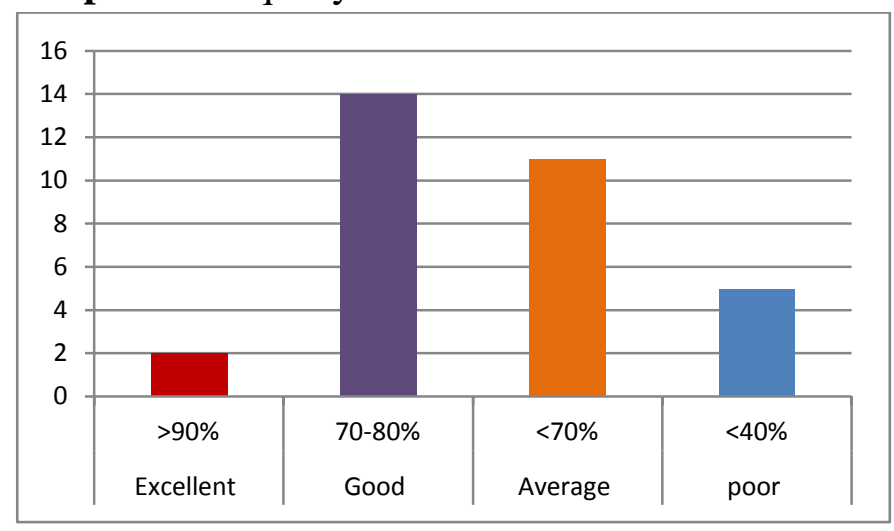

In the Prone position, colonic distension was good for the entire colon except for transverse colon, while in supine position descending and ascending colon was average and good distension was achieved for transverse colon, rectum and sigmoid colon.

\section{Discussion}

Colorectal carcinoma is the 3rd most common cancer in the men and 2nd most common among women ${ }^{[8]}$. With changing dietary habits towards junk food and less fiber-containing whole natural foods the incidences of colon carcinoma are increasing in the population. Hence it forms an important burden on the well being of the society. Early detection of the colonic pathologies and premalignant conditions go a long way in reducing this burden of disease and reduce the morbidity and mortality. The current study included $n=32$ patients with a mixed composition of colonic symptoms undergoing CT Colonography screening. Among the $n=32$ patients, malignancy was detected in $\mathrm{n}=6$ cases (18.7\%) and 67 percent of the carcinomas were in the distal colon. Among the $n=6$ cases of carcinoma colon, $\mathrm{n}=4$ cases had advancing carcinoma beyond the colonic wall. Among the $\mathrm{n}=4$ cases 50 percent $\mathrm{n}=2$ cases presented with metastasis to distal organs. Kim et al $;^{[9]}$ of 241 patients in a single center in a screening setting with cathartic preparation devoid of any kind of fecal tagging in 2D mode detected only one case of colonic malignancy by CT Colonography. While in our study is a tertiary center we had a mixed set of cases some of which have been diagnosed outside, hence the high percentage in our study. Ozsunar $\mathrm{Y}$ et al; ${ }^{[10]}$ studied $\mathrm{n}=48$ cases in the non-screening setting without fecal tagging concluded with $n=4$ cases of colonic malignancy. This study correlates with our study. Advanced carcinomas presented in this study because of the poor awareness of the patients selected from poor socio-economic conditions that came when the symptoms went advanced, whereas among western patients high awareness regarding the presenting symptoms and high availability of doctors as well as CT scanner per patient is high. According to Pickhardt PJ et al; ${ }^{[11]}$ Residual stool represents a fundamental diagnostic challenge for CTC interpretation, even when cathartic agents are employed. In this study, we tried to differentiate stool versus growth or polyp based on the morphology. Almost all cases had some kind of residual stool adherence to the intraluminal wall and it became even more difficult when the colon was incompletely distended in places or sub-optimally distended with poor bowel evacuation. Few cases of falsepositive polyps identified which on $\mathrm{VC}$ failed to demonstrate suggesting that complete bowel preparation is quite essential for a good study. Pickhardt PJ et al $;^{[11]}$ while fecal tagging and digital cleansing are new evolving techniques this study had these limitations. This study has many cases with significant bowel fluid as we had the colonic preparation done with different strategies based on the availability of the pharmaceuticals. But preparations with Sodium Picosylate were superior to polyethylene glycol or Soap water enema. Patients who were asked to take polyethylene glycol failed to take an adequate amount of water as advised hence evacuation was 
inferior to Sodium Picosylate, while Soap water enema prepared subject had significant colonic fluid at the time of the study. Overall Sodium picosulfate was superior to either of the two correlating with Macari et al; ${ }^{[12]}$ and Mang $\mathrm{T}$ et al; [13]. In our study involved organs are vascular system (21.8\%), the genitourinary system $(42.8 \%)$, the liver $(15.6 \%)$, the lymphatic system $(0.03 \%)$, the lungs $(0.03 \%)$, and the gynecological system $(0.03 \%)$. Only $n=4$ cases had potentially significant findings with malignancy contributing $12.5 \%$ which includes metastasis and lymphoma. According to studies a new technique for assistance to the radiologist with a second opinion $^{[14-17]}$. This is done by software algorithms that automatically highlight polyps and abnormal growth while differentiating from insignificant projections into the lumen. Current systems having a sensitivity of up to $90 \%$ in polyps of size 0.5 to $2 \mathrm{~cm}$. CAD can be used by the radiologist as assistance but not for diagnosis because CAD will be misled by a wide range of pseudolesions such as residual stool, mucus, and impacted diverticula; motion artifacts or spasms; intrinsic bowel structures such as the ileocecal valve and bulbous folds; the rectal tip; and the extracolonic lesions in the small bowel or stomach.

\section{Conclusion}

Virtual colonoscopy was found to be very useful because of 3D viewing of colon both in forward as well as reverse directions. However, it has limitations because it depends on proper bowel preparation. Electronic bowel cleansing or barium fecal tagging may improve sensitivity. This technique is very useful in elderly patients where sedation is contraindicated and also useful in patients with Diverticulosis where chances of perforation are high by conventional colonoscopy.

\section{References}

1. EJ Kuipers, Willam MG, David L, Thomas $\mathrm{S}$, et al. Colorectal Cancer. Nat Rev Dis Primers 2015; 1: 15065.
2. ICMR Subcommittee on Colorectal Cancer. CONSENSUS DOCUMENT FOR MANAGEMENT OF COLORECTAL CANCER. available online. https://www.icmr.nic.in/sites/default/files/ guidelines/Colorectal\%20Cancer_0.pdf [Accessed on 12/12/2019]

3. Muto T, Bussey HJ, Morson BC. The evolution of cancer of the colon and rectum. Cancer 1975; 36:2251-2270.

4. Winawer SJ, Zauber AG, Ho MN, et al. Prevention of colorectal cancer by colonoscopic polypectomy. The National Polyp Study Workgroup. N Engl J Med 1993; 329:1977-1981.

5. Muller AD, Sonnenberg A. Prevention of colorectal cancer by flexible endoscopy and polypectomy. A case-control study of 32,702 veterans. Ann Intern Med 1995; 123:904-910.

6. Thiis-Evensen E, Hoff GS, Sauar J, Langmark F, Majak BM, Vatn MH. Population-based surveillance by colonoscopy: effect on the incidence of colorectal cancer. Telemark Polyp Study I. Scand J Gastroenterol 1999; 34:414-420.

7. Citarda F, Tomaselli G, Capocaccia R, Barcherini S, Crespi M. Efficacy in standard clinical practice of colonoscopic polypectomy in reducing colorectal cancer incidence. Gut 2001; 48:812-815.

8. Abhishek Bhandari, Melissa Woodhouse, Samir Gupta. Colorectal cancer is a leading cause of cancer incidence and mortality among adults younger than 50 years in the USA: a SEER-based analysis with comparison to other young-onset cancers. J Investig Med 2017; 65(2):31115.

9. Kim YS, Kim N, Kim SH, et al. The efficacy of intravenous contrast-enhanced 16-raw multidetector CT colonography for detecting patients with colorectal polyps in an asymptomatic population in Korea. J Clin Gastroenterol 2008; 42(7): 791 - 798. 
10. Ozsunar Y, Coskun G, Delibaş N, Uz B, Yükselen V. Diagnostic accuracy and tolerability of contrast-enhanced CT colonoscopy in symptomatic patients with increased risk for colorectal cancer. Eur $\mathbf{J}$ Radiol 2009; 71 ( 3 ): 513 - 518.

11. Perry J. Pickhardt, MD and David H. Kim. CT Colonography. Pitfalls in Interpretation. Radiol Clin North Am. 2013; 51(1): 69-88.

12. Macari M, Bini EJ, Jacobs SL, Lange N, Lui YW. Filling defects at CT colonography. Radio Graphics 2003;23:1073-91.

13. Thomas Mang, Andrea Maier, Christina Plank, Christina Mueller-Mang, Christian Herold, Wolfgang Schima. Pitfalls in Multi-Detector Row CT Colonography. RadioGraphics 2007; 27:431-454.

14. Summers RM, Yao J, Pickhardt PJ, et al. Computed Tomographic virtual colonoscopy computer-aided polyp detection in a screening population. Gastroenterology 2005;129:1832-1844.

15. Yoshida H, Nappi J, Mac Eneaney $P$, Rubin DT, Dachman AH. Computer-aided diagnosis scheme for detection of polyps at CT colonography. Radio Graphics 2002;22:963-979.

16. Taylor SA, Halligan S, Burling D, et al. Computer-assisted reader software versus expert reviewers for polyp detection on CT colonography. AJR Am J Roentgenol 2006;186:696-702.

17. Summers RM, Jerebko AK, Franaszek M, Malley JD, Johnson CD. Colonic polyps: complementary role of computer-aided detection in CT colonography. Radiology 2002;225:391-399. 